\title{
Teatro gesuita e tradizione pagana: due scenari di argomento polacco nella Francia di Mazarino
}

\section{Simona Munari}

\section{(2) OpenEdition}

\section{Journals}

Edizione digitale

URL: http://journals.openedition.org/studifrancesi/27058

DOI: 10.4000/studifrancesi.27058

ISSN: 2421-5856

Editore

Rosenberg \& Sellier

\section{Edizione cartacea}

Data di pubblicazione: 31 décembre 2006

Paginazione: 524-533

ISSN: 0039-2944

\section{Notizia bibliografica digitale}

Simona Munari, «Teatro gesuita e tradizione pagana: due scenari di argomento polacco nella Francia di Mazarino », Studi Francesi [Online], 150 (L | III) | 2006, online dal 30 novembre 2015, consultato il 08 novembre 2020. URL : http://journals.openedition.org/studifrancesi/27058 ; DOI : https://doi.org/ 10.4000/studifrancesi.27058

\section{(c) (i) $\odot$}

Studi Francesi è distribuita con Licenza Creative Commons Attribuzione - Non commerciale - Non opere derivate 4.0 Internazionale. 


\section{Teatro gesuita e tradizione pagana: due scenari di argomento polacco nella Francia di Mazarino}

Nel 1646, per i tipi di Toussainct Quinet, viene pubblicata a Parigi una tragicommedia di argomento polacco, il Sigismond duc de Varsau di Gillet de la Tessonerie. Gillet, autore di commedie e tragicommedie di successo, rielabora una materia che si stava diffondendo in Francia per motivi di carattere essenzialmente politico. Si poneva infatti il problema di celebrare sulla scena letteraria l'inizio del lungo regno (fino al 1668) di Luisa Maria Gonzaga, andata sposa a Ladislao IV nel 1645 sotto l'auspicio del cardinal Mazarino. Il matrimonio contribuì a rafforzare i rapporti tra Francia e Polonia, esistenti sin da quando la candidatura di Henri de Valois era stata proposta nel 1573 alla dieta polacca per ricoprire il trono vacante. Da quel momento si era moltiplicata la divulgazione di relazioni diplomatiche e resoconti di viaggio in cui la Polonia era presentata come una terra promessa, ricca e generosa, dotata di «humanité et douce conversation». Si insisteva sulle somiglianze con gli usi e costumi francesi, e sulla posizione geografica che faceva della Polonia l'ultima nazione civilizzata, baluardo della cristianità. Il fasto della sua corte si prestava inoltre a facili esotismi, il più forte dei quali riguardava la diversità delle istituzioni politiche, soprattutto in relazione alla modalità di scelta del sovrano ${ }^{1}$.

Venda regna en l'an 750 et quitta volontairement la couronne, après avoir gaigné une bataille contre un de ses sujets, qui vouloit la contraindre à l'aymer.

Histoire de Pologne.

Gillet pone questa precisazione nell' elenco dei personaggi, a margine del nome dell' eroina ${ }^{2}$. Narra la leggenda che Vanda (Wanda, Vende), figlia del re Krak o Cracus, fondatore e primo sovrano di Cracovia, fu eletta regina dal popolo dopo la morte del padre. Il fratello minore di Vanda aveva infatti assassinato l'erede al trono ed era poi morto di dolore. La giovane principessa governa il paese votando la sua verginità agli dei per ottenere la loro protezione, rifiuta tutti i pretendenti e sconfigge il principe tedesco Ritiger quando costui cerca di appropriarsi del regno. Consegue la vittoria senza battaglia, giacché i nemici si rifiutano di lottare contro una donna, e il loro capo si uccide. Ma al rientro a Cracovia, in luogo di ricevere gli onori dei sudditi, Vanda si lancia nella Vistola come supremo sacrificio agli dei che hanno salvato il paese. Gli annali di Boemia riportano storie simili (in particolare la leggenda di Libusa, figlia del re $\mathrm{Krok}^{3}$ ), che già presentano le linee tematiche dalle quali ha preso forma l'immagi-

(1) Tra gli autori inclini all'ispirazione polacca F. Rosset cita, insieme al Gillet del Sigismond, Rotrou (Venceslas), Camus (Iphigène), Préchac (Le Beau Polonois), oltre a opere anonime, come il romanzo Le Polemire ou l'illustre Polonois, sottolineando come i motivi polacchi, inizialmente privilegiati per la coloritura esotica, assumano ben presto una complessità simbolica. F.RossET, L'Arbre de Cracovie. Le mythe polonois dans la littérature française, Imago, Paris, 1996, pp. 63-66. Cfr. anche gli atti del convegno La France et la Pologne. Histoires, mythes, représentations, éd. F. Lavocat, Lyon, Presses Universitaires de Lyon, 2000.
(2) Gillet de la Tessonerie, Sigismond duc de Varsau, Paris, Quinet, 1646.

(3) Il re Krok ha tre figlie. Prima di morire passa il potere alla minore, Libusa, la cui intelligenza e magnaniminità ben si coniugano alla bellezza. Istigata dal popolo che vuole essere governato da un uomo, Libusa sposa il saggio contadino Premislas, col quale governa felicemente e fonda la città di Praga. Alla morte della regina un gruppo di ragazze capeggiate da Wlasta tenta di impadronirsi del potere: scoppia una guerra nella quale le truppe di Premislas faticano ad avere la meglio sulle amazzoni. F. Rosset, Wanda: 
nario mitico su cui poggiano le trasposizioni letterarie: la religiosità pagana, la lotta fratricida, il potere femminile, la fondazione della città, il suicidio-sacrificio.

La storia complicata proposta da Gillet, assai diversa dalla leggenda originale, sembra derivare almeno in parte dal teatro. Per quanto, infatti, quella tradizione potesse essere nota in Francia, è dubbio che le cronache polacche siano state le fonti dirette dei letterati francesi. Nella sua accurata indagine Daniela Dalla Valle ha messo in relazione la breve vicenda polacca di Henry de Valois con la pubblicazione in Francia di opere destinate a illustrare un paese quasi sconosciuto: la storia di Vanda compare nella Cosmographie di Munster e nelle traduzioni francesi di Bauduin e di Vigenère delle cronache di Fulstin, poi nel breve Discours sur l'histoire des Polognois attribuito a Nicolas Pavillon; in seguito, fu ripresa da Pierre Davity e da Jean Le Laboureur ${ }^{4}$. Al di là di alcune varianti sulla datazione, gli elementi più significativi (la morte dei fratelli, l'elezione della regina, le sue doti fisiche e morali, la conclusione tragica) non sono modificati. Il suicidio è però presentato secondo una prospettiva edificante che la studiosa mette in relazione agli «erreurs» compiuti da un popolo nel nome di false credenze, sottolineando come la dimensione religiosa, che, vede nel sacrificio della regina un'impresa di purificazione, informi anche i primi interventi letterari, due tragedie latine di collegio di cui sono pervenuti soltanto gli scenari: La piété polonaise ou Venda Reine de Pologne «che si consacra ai suoi dei» recitata il 3 agosto 1639 al collegio di Rouen e forse il 5 agosto 1643 al collegio parigino Louis-le Grand, e Vende, ou le Triomfe et le sacrifice de la chasteté, rappresentata a Troyes nel $1644^{5}$.

L'attività pedagogica della Compagnia di Gesù, basata sulla Ratio studiorum, prevedeva l'impegno degli allievi in rappresentazioni teatrali di argomento sacro o comunque improntato alla pietà, allo scopo di «ad perfectam eloquentiam pervenire». La pratica degli spettacoli, destinati all'inizio a celebrare particolari momenti della vita scolastica, come le premiazioni pubbliche, fu presto allargata a molte occasioni festive: canonizzazioni, consacrazioni di chiese, giubilei, matrimoni principeschi. Le pièces erano redatte dai professori di retorica e rappresentate dagli studenti, con l'ausilio di un choragus, mentre la stesura dell'argomento, o scenario, era di solito affidata a un convittore ${ }^{6}$. I testi teatrali erano scritti in latino, lingua in cui per secoli si era espressa la cultura europea, ma nei collegi diretti dai Gesuiti lo studio della lingua francese fu attivato sin dalla metà del Seicento. Non si può quindi escludere che di alcune pièces si desse volontariamente notizia in volgare, come nel caso delle tragedie di argomento polacco, dal momento che il francese si apprestava a diventare la lingua europea di cultura ${ }^{7}$. D'altronde il teatro secentesco - ricorda M. Fumaroli

du mythe au roman, «Dix-huitième siècle», 27, 1995 , p. 456.

(4) S. Munster, La Cosmographie universelle (1556); B. DE VIGENĖRE, Les Chroniques et annales de Poloigne (1573); J.H. DE Fulstin, Histoire des Roys et princes de Poloigne (1573); N. PaVILlon, Discours sur l'bistoire des Polognois (s.d.); P. DAvity Les Etats empires royaumes et principautez du monde (1625); J. Le Laboureur, Histoire succinte par éloges, de tous les Princes et Rois de Pologne in Histoire et Relation du voyage de la Reine de Pologne (1648). Maggiori informazioni su questi testi in D. Dalla Valle, Autour de "Sigismond, duc de Varsau" de Gillet de la Tessonerie. $L$ 'bistoire et le mythe de Vanda en France, in La France et la Pologne. Histoire, mythes, représentations, éd. F. Lavocat, Lyon, Presses Universitaires de Lyon, 2000, p. 183.
(5) F. Rosset considera la tragedia del 1644 fonte diretta della tragicommedia di Gillet (cfr. Wanda, du mythe au roman, cit., p. 457); in seguito D. Dalla Valle ha messo in relazione il Sigismond anche alla tragedia del 1639, descrivendo entrambi gli scenari nell'articolo Autour de "Sigismond duc de Varsau”, de Gillet de la Tessonerie, cit., pp. 185-186.

(6) J.M. Valentin, Les jésuites et le théâtre (1554-1680), Paris, Desjonquères, 2001, pp. 101116 e Les jésuites et la scène: Orphée, Pallas et la renovatio mundi, in Les jésuites à l'âge baroque, cit., pp. 131-142.

(7) Cfr. G. P. Brizzi, La formazione della classe dirigente nel Sei-Settecento, Bologna, Il Mulino, 1976, p. 239-240. 
- era inserito in una retorica generale in cui l'attore-oratore, con la voce e i gesti, si proponeva come principale motore dell'azione drammatica in cui il testo è un ausiliario importante, ma secondario ${ }^{8}$. Non è quindi escluso che gli scenari - presentazioni che descrivevano eventuali scelte compositive dell'opera, ambientazioni sceniche e azioni dei personaggi, insieme alla lista completa degli attori - avessero una diffusione maggiore rispetto ai testi veri e propri.

Lo scenario della Piétépolonoise (1639) comprende una sorta di riassunto in versi diviso per atti, un breve «argument de la tragédie», e un elenco preciso dei «noms des acteurs et leurs personnages» diviso per ruoli: i polacchi, i tedeschi, il coro «à la façon des anciens», le guardie reali, i pastori e i Geni del prologo, e poi la Vittoria, l'Onore, la Fama, la Morte. La vicenda è riferita in questi termini: Vanda promette a Sivare conte di Varsavia di sposarlo se la aiuta nella guerra contro il re dei Teutoni Molonte. Quando Sivare viene sconfitto, la regina chiede aiuto agli dei in cambio di «ce qu'ils auroient de plus cher en son Royaume». Al momento della successiva vittoria, Vanda teme di dover sacrificare il conte, e per evitarlo consulta nuovamente gli dei, i quali la indicano come vittima designata. Si getta allora nel fuoco sull' altare, dove Sivare disperato vorrebbe seguirla. La Vistola, per l'orrore, si ritira alla sorgente:

l'impassibilité de tous les elements éprouve en cet instant les plus doux sentiments?.

L'argomento di Vende ou le triomfe et le sacrifice de la chasteté (1644), più articolato del precedente, comprende anche una dedica al marchese di Praslain, governatore di Troyes, un Avertissement ai lettori e un riassunto per scene dei cinque atti: la principessa accetta di essere eletta alla morte del padre, e riceve la proposta di matrimonio del duca sassone Ritiger. Respinto, costui chiede aiuto a un mago, che gli dà un anello magico. L'amore dura finché l'anello non cade, e allora Ritiger cerca di conquistarla con le armi, ma fallisce e si uccide. La regina si immola alla gloria divina lanciandosi in un precipizio ${ }^{10}$.

L'obiettivo edificante passava per una forma barocca di rappresentazione, ideale ricalco dei fasti di corte e delle processioni cui lo spettatore era avvezzo, che aveva nell'elemento visivo (aspetti musicali e scenografici) una componente fondamentale, a compensazione dell'ostico latino dei dialoghi. Il modello della tragedia senecana si coniugava felicemente a una spettacolarità fatta di colpi di scena, apparizioni, intermezzi, allegorie ${ }^{11}$, ma il gusto francese, fedele allo spirito umanista della seconda metà del Cinquecento, tendeva a scansare il patetico melodrammatico e la tragedia biblica a favore di storie esemplari: eroi impegnati in un'azione umana, spesso a sfondo storico, che impone loro di compiere scelte difficili ${ }^{12}$.

(8) M. Fumaroli, Eroi e oratori, Retorica e drammaturgia secentesche, 1990 , tr. it.: Bologna, Il Mulino, 1990, p. 21.

(9) La Piété polonaise ou Venda Reine de Pologne, qui se consacre à ses dieux. Pour la distribution des prix de Monseigneur Messire Nicolas Turgot, Sieur de Lantueuil, etc. Conseiller du Roy en ses Conseils, et President au Parlement de Normandie, Rouen, Jean le Boullenger, près le College des PP. Jésuites, 1639.

(10) Vende, ou le triomphe et le sacrifice de la chasteté. Tragedie. Representée au Collège des PP. De l'Oratoire de Jesus à Troyes, Troyes, Jean Jacquard, 1644.

(11) Nella seconda metà del Seicento la maggior parte dei collegi fu dotata di teatri, i cui apparati scenografici raggiunsero un'elevata perfezione tecnica che rispondeva al gusto del pubblico e ad esigenze di carattere propagandistico. Cfr. G.P. BRIZZI, op. cit., pp. 249-250 e F. DE DAINVILle, L'éducation des jésuites (XVI ${ }^{e}$-XVIII ${ }^{e}$ siècles), Paris, éd. de Minuit, 1978, pp. 476-503.

(12) A. Stegmann, L’héroïsme cornélien, génèse et signification, II, Paris, Colin, pp. 51-52 e E. WE$\mathrm{BER}$, Le théatre humaniste protestant à participation musicale et le théâtre jésuite: influences, convergences, divergences, in G. Demerson et al. (c.d.), Les jésuites parmi les hommes aux XVI et XVII siècles, cit., pp. 445-460. 
Vende ou le triomfe et le sacrifice de la chasteté - si legge nella dedica al marchese di Praslain - è

le portrait d'une royale amazone, qui croit que ce qui n'est pas connu à la France est dans le malheur commun des choses cachées aux siècles à venir; qui veut que son nom aussi bien que ses rares vertus peu connues parmi nous mais fameuses dans les histoires polonaises soient autant signalées chez une nation qui fait gloire de lui avoir donné ses rois pour successeurs de la couronne, qu'elles sont en vénération parmi les peuples qui l'ont veue, mais trop peu, si vertueusement régner; reine qui s'aquittant des actions des plus hardis guerriers a fait voir aux femmes de son temps et appris par son histoire à la postérité que le sexe ne met point d'obstacle à l'ardeur d'un grand courage lorsqu'il est question de pousser un généreux dessein jusques où il peut être conduit.

La scuola gesuitica tedesca e quella francese postulavano la superiorità della verosimiglianza rispetto al vero, ammettendo l'adesione soltanto parziale alle regole del tragico antico. Ne troviamo riscontro nell'Avertissement di Vende ou le triomfe et le sacrifice de la chasteté:

J'ai voulu contenter également tout le monde, en faisant que toute sorte de personnes fût capable de l'entendre. Au reste, Messieurs, comme il n'est pas ici question de vous montrer notre suffisance, mais de vous prouver seulement l'assiduité de nos soins à exercer votre jeunesse, je crois que vous ne me blâmerez pas d'avoir pris un sujet où vous aurez l'avantage de juger des actions de vos enfants.

La grandezza dell'individuo si afferma nella lotta e nell'avversità, si misura per contrasto con l'invidia e la gelosia, la sete di potere e ricchezze, il desiderio impuro, la menzogna, il tradimento, in un messaggio di semplice decodifica e ricezione. Lo schema è articolato in due fasi: confronto dell'eroe con ostacoli di ogni natura e modalità del suo rapporto con gli altri personaggi, in un ampio ventaglio di caratteri, passioni, virtù e vizi che sottolineano l'importanza del rigore morale sino ad evocare posizioni neo-stoiche ${ }^{13}$. Il sistema dei teatri di collegio, con le sue modalità espressive che ricoprivano un posto di rilievo nella pratica pedagogica, imponeva la revisione delle regole in nome della pietas litterata, e l'inserimento in una dimensione cristiana di personaggi appartenenti a tradizioni culturali estranee.

Pensiamo ad esempio al Sigismondo, tragedia latina che evoca però solo nel titolo la materia di cui ci occupiamo, rappresentata nel 1617 al Seminario Romano: il compositore fa «calzare in molta fretta i coturni» al re di Borgogna Sigismondo, ricamando «con agio poetico» una vicenda ambientata ai tempi di Clodoveo ${ }^{14}$. Fatti salvi i diversi contesti politici e letterari dei paesi nei quali i gesuiti operavano, la rivendicazione di spiritualità passava per argomenti storici e allegorici, e soprattutto per la cristianizzazione di motivi e figure presi a prestito dal mondo greco e romano. Se consideriamo la diffusione europea della Compagnia di Gesù, e il fatto che gli altri ordini religiosi non si discostarono sostanzialmente dai criteri educativi contenuti nella Ratio Studiorum - precisa G.P. Brizzi - ben si comprende come tra il Cinquecento e il Settecento si sia determinata un'unità nell'indirizzo pedagogico che favorì la formazione di una classe dirigente europea caratterizzata da comuni matrici educative ${ }^{15}$.

In questa prospettiva fu senz'altro determinante l'opera del gesuita polacco Maciej Kazimierz Sarbiewski, l'Horatius sarmaticus, autore di raccolte in versi che

(13) M.J. Valentin, Les jésuites et le théâtre (1554-1680), cit., pp. 156-185.

(14) Cfr. B. FILIPPI, Il teatro degli argomenti. Gli scenari seicenteschi del teatro gesuitico romano, Roma, Institutum historicum S.I., 2001, pp. 79-83.

(15) G.P. BRIzzI, op. cit., p. 18 
lo resero celebre come poeta cristiano e teorico della creazione poetica ${ }^{16}$. Allievo dei gesuiti polacchi ma formatosi a Roma tra il 1622 e il 1625, era professore di retorica, fisolofia e teologia a Vilna, e fu inviato alla corte di Varsavia nel 1635. Quasi certamente aveva incontrato Ladislao Sigismondo in Italia: come molti giovani regnanti in tempi di Controriforma, il principe svedese di Polonia viaggiava per ampliare la sua formazione frequentando gli ambienti artistici più significativi, e tra il 1622 e il 1625 era stato accolto con tutti gli onori alla corte medicea. Divenuto re nel 1632, aveva introdotto nel suo paese la cultura spettacolare italiana, facendo costruire da Agostino Locci il Teatro Reale di Varsavia e rendendo possibili brillanti stagioni musicali curate da Virgilio Puccitelli, il suo segretario marchigiano ${ }^{17}$.

A partire dal 1564 l'attività dei Gesuiti in Polonia, grazie alla protezione del re Sigismondo III (1587-1632), era stata molto intensa: nonostante le difficoltà, nel 1634 la Compagnia dirigeva venticinque collegi, con un effettivo di un migliaio di religiosi, e fino al 1648 registrò in campo educativo uno sviluppo costante, ponendosi come riferimento di contenuti e metodi nonostante l'avversione della vecchia università, appoggiata dalle sedi di Padova e Lovanio ${ }^{18}$. I professori stranieri furono man mano sostituiti dagli allievi polacchi, molti dei quali appartenevano a famiglie influenti e si erano convertiti, come i figli di Nicolas Radziwill (1515-1565), capo dei calvinisti lituani ${ }^{19}$. Fino alla crisi di metà Seicento l'attività gesuitica in Polonia è un ulteriore elemento che giustifica l'interesse per la storia e le leggende di quei luoghi, già noti in Francia per motivi legati all'attualità storica. Le tragedie di collegio che hanno Vanda come protagonista, rispondendo a un evidente imperativo di edificazione morale assente dalle rielaborazioni successive ${ }^{20}$, costituivano un'occasione ideale per unire

(16) Alcuni anni dopo il soggiorno romano scrisse il trattato De perfecta poesi, sive Vergilius et Homerus, in cui descrive il sistema scenografico visto all'opera nel Collegio Romano. Su Mathias Casimir Sarbievius, 1595-1640, cfr. J.M. VALENTIN Le théatre des collèges de la fin du XV siècle à la fin du XVIII', in Dictionnaire de spiritualité, t. XV, Paris, Beauchesne, 1990-1991, p. 355; G. ZANLONGHI, Teatri di formazione. Actio, parola $e$ immagine nella scena gesuitica del Sei-Settecento a Milano, Milano, Vita e Pensiero, 2002, pp. 226-227; C. Sommervogel, Bibliotbèque de la Compagnie de Jésus, Louvain, éd. de la Bibliothèque S.J., Collège philosophique et théologique, p. 627; B. FiLIPPI, Il teatro degli argomenti, cit., p. 56.

(17) S. Carandini, Teatro e spettacolo nel Seicento, Roma-Bari, Laterza, 1990 , pp. 38-39.

(18) A. JoberT, De Luther à Mobila. La Pologne dans la crise de la Chrétienté (1517-1648), Paris, Institut d'études slaves, 1974, p. 259.

(19) Il maggiore, Nicolas (1549-1616), fondò il collegio gesuita di Nieswietz, mentre il giovane Georges (1556-1600), chiamato da Gregorio XIII a seguire i corsi del Collegio Romano, divenne vescovo di Wilno e di Cracovia. Cfr. W. MüLler, Les jésuites en Pologne aux XVI et XVII siècles, in G. DeMERson et al. (éd.), Les Jésuites parmi les bommes aux XV et XVII siècles, Clermont-Ferrand, Faculté des Lettres et des Sciences humaines, 1987, pp. 323-330.

(20) Dopo Gillet, il teatro profano celebra la storia di Vanda nella tragedia di Jobert, Balde reine des Sarmates (1651), in cui cambiano solo i nomi dei protagonisti: la regina si suicida dopo aver vinto in battaglia Trasonte, col quale aveva rifiutato di sposarsi per amore di Voltare, osteggiato dal ministro Adolphe e dal capitano Rutile. Un duello fra i pretendenti pare risolutivo, ma nel momento in cui Voltare sta per essere sconfitto dal rivale, Balde salva il regno offrendo agli dei ciò che ha di più caro: per mantenere il voto, Voltare si dispone a morire, cedendo la mano di Balde al rivale, invece è lei a gettarsi nella Vistola. Nel secolo successivo Vanda diventa la protagonista di una «nouvelle romanesque et galante» anonima la cui fonte principale sembra essere la tragicommedia di Gillet. In Vende Reine de Pologne ou l'bistoire galante et curieuse de ce qui s'est passé de plus memorable en ce temps-là (La Have, 1705), sono due amiche di Vende, Casimire e Thorixenne, a narrare l'episodio che tradisce la conoscenza delle fonti storiche da parte dell'autore: i conflitti tra i fratelli della regina prima dell'elezione assumono però un risvolto sentimentale e incestuoso, e l'innamorato di Vanda si chiama Premislas, indicato come suo successore nelle cronache polacche tradotte in francese. L'editore della novella precisa di avere «un peu adouci l'evenement», e infatti Vanda, dopo essersi suicidata nella Vistola, viene ripescata dal prete del tempio, e sposa Premislas che nel frattempo ha salvato il regno. Il ritorno della materia polacca al teatro avviene nel 1751 con la tragedia di Linant Vanda, reine de Pologne (Paris, Cailleau), in cui Premislas assume il ruolo di difensore in un'intricata vicenda di guerre e amori molto lontana dalla leggenda originale. Un'analisi puntuale delle rielaborazioni letterarie della leggenda polacca è proposta da Daniela Dalla Valle nell'articolo Autour de "Sigismond", cit. 
il fine religioso a una bellezza nutrita di antichità, posto che la vicenda fosse riletta, sin dal titolo, in chiave di «piété». Su un piano più strettamente tematico, la storia di Vanda associa al motivo della fondazione della città-capitale la questione del potere femminile, e il mito ad esso collegato, quello dell'amazzone ${ }^{21}$.

Resta aperto il problema del suicidio: gli storici francesi lo ritenevano un fattore di inverosimiglianza che poteva pregiudicare la comprensione dell'intera vicenda, e cercarono spiegazioni politiche, psicologiche, mediche. Rosset ricorda le interpretazioni dell'abate Desfontaines (Histoire des révolutions de Pologne, 1735), che addebita il triste esito della vicenda alla follia della regina, e quella di Contant d'Orville (Les fastes de la Pologne et de la Russie, 1770) incline a considerare il sacrificio una conseguenza dell'orgoglio. La necessità di non tradire un voto pubblico di verginità è anche la chiave di lettura di A. Homot (Anecdotes du Nord, 1770) ${ }^{22}$, ma permane di fatto irrisolta una questione che appare centrale nel determinare il percorso di ricezione in Francia della materia polacca. Se infatti riteniamo valida la filière che vede nelle tragedie gesuitiche l'origine della sua fortuna letteraria, diventa importante capire per quale motivo il teatro di collegio ha riproposto nelle due pièces in esame un finale tanto cruento. Il limite di avere a disposizione soltanto gli scenari, e di non poter quindi consultare i testi, costringe l'analisi entro margini molto stretti. A una prima valutazione, appare indiscutibile che la morte dell' eroina per suicidio esprima la voce della "moralità" pedagogica di cui il teatro gesuitico si faceva interprete: Vanda si vota agli dei pagani, dunque non può essere salvata, nemmeno in extremis. Nella tragedia del 1639 si legge infatti:

Elle prend la blancheur d'une innocente hostie,

Et vouant à ses Dieux Sivare, et ses subiets,

Elle esleve sa veue aux Celestes obiets,

Son courage animé d'une divine flame,

Ne peut plus retenir les transports de son ame,

Elle invite le Prestre à ne prolonger plus,

D'un miserable estat les momens superflus,

Elle addresse son bras dessus un sein d'albastre,

Ai lieu de la frapper son bourreau l'idolatre,

En fin le souvenir des Dieux et de son rang,

L'oblige de tremper le cousteau dans son sang ${ }^{23}$.

«La Reine a fait un voeu pour ce qui depend d'elle» - è precisato nel riassunto del quarto atto, ma il voto agli dei pagani garantisce la salvezza della città, non un destino felice:

Puisque la fortune a trahi les desirs,

Elle veut que sa mort couvre ses desplaisirs,

Et malgré son destin n'ayant pu vivre heureuse,

Elle s'asseure au moins de mourir genereuse.

Nella seconda tragedia (1644) la dedica sottolinea «la prudence dans ses affaires, la constance à mespriser l'Amour, et l'invincible Courage à punir les flâmes d'un Prince indiscret et trop excessivement passionné pour sa Beauté»: la regina rifiuta il

(21) Jaucourt focalizzò il suo articolo Pologne nell'Encyclopédie sulla questione del potere femminile, scrivendo che all'epoca di Vanda l'Oriente aveva già deciso che la donna era nata per ubbidire, ma la principessa potè regnare a lungo in quanto la Polonia ancora non aveva adottato quel sistema.

Cfr. F. Rosset, L'arbre de Cracovie, cit., p. 457.

(22) F. Rosset, L'arbre de Cracovie, cit. p. 457.

(23) La piété polonaise ou Venda reine de Pologne, cit., cinquiesme acte. 
matrimonio, in linea con un'impostazione rigorosa nel salvaguardare i valori della religione, e difende la castità con la morte, ma già si nota la presenza di un voto ambiguo che potrebbe provocare la perdita dell'uomo amato ${ }^{24}$, e al suicidio è riservato appena un cenno nel riassunto del quinto atto:

Et Vande [...] pour recognoistre ses Dieux de tous ses Triomphes, se va immoler à leur Gloire dans un precipice ${ }^{25}$.

Questa versione, in cui il suicidio è agito mediante il lancio nel precipizio, sarà mantenuta nelle rielaborazioni successive. Nell'bistoire galante et curieuse del 1705 (Venda Reine de Pologne) l'eroina viene ripescata dal fiume e nascosta in un tempio: se qui l'opzione del lieto fine appare legata al rigido sistema di regole che vincolava il genere romanzesco, è però vero che il motivo del salto prodigioso - ovvero non mortale - era diffuso sin dal Medioevo. La vittima era raccolta "in volo", come la bella Alda che si lancia dalla rocca di Susa per sfuggire ai Saraceni (ma quando si vanta di essere stata raccolta da un angelo e riprova, si schianta al suolo), o sopravviveva miracolosamente alla caduta, come Santa Idda, moglie innocente gettata da una torre per ordine del marito geloso. La protagonista della novella Venda Reine de Pologne ferma la mano dei sacerdoti che stanno per immolare cento animali agli dei, annunciando di voler cercare l'immortalità nella morte ${ }^{26}$, ma solo alla fine, secondo gli schemi ricorrenti nella prosa francese, l'amato Premislas la ritrova, proprio nel momento in cui sta per gettarsi a sua volta, disperato, nel fiume. La vicenda si conclude con l'agnizione e il matrimonio. Paradossalmente, il potere taumaturgico dell'acqua sembra compiere il "miracolo", e il tratto pagano viene rivisitato in chiave cristiana con l'introduzione di quel gesto al quale nelle Vitae e nei Miracula medievali ci si riferiva come «sauvetage des chutes» ${ }^{27}$.

Nel caso delle tragedie gesuitiche, si assiste forse ad una rievocazione indiretta del topos dantesco del sacrificio della vita in nome di un valore più alto, ma gli elementi in nostro possesso tendono piuttosto a dipingere il suicidio come un «récit de châtiment», una sorta di castigo divino, una forma estrema di altari sancti se offerre: Vanda deve onorare un voto di verginità, e poiché per un momento è tentata dall'amore, si uccide per espiare quel desiderio, e non soltanto per ringraziare gli dei di avere salvato il regno. Non è escluso che il teatro gesuita intendesse farsi portavoce di un'attitudine rigorista nei confronti del «marchandage» miracolistico che sin dal Medioevo animava una parte della Chiesa ${ }^{28}$ : secondo questa linea più rigida, l'intervento divino non era legato all'espressione di un voto, ma a una serie di circostanze svincolate dal controllo umano. Per quanto la protezione dalle catastrofi e dai pericoli collettivi rientrasse nella casistica ufficiale della tipologia di voto, il vacillare di Vanda di fronte alla morte, o meglio il scegliere la morte come male minore rispetto a una vita senza amore, poteva essere letto - al di là della sua appartenenza religiosa - come una perdita di fede, e pertanto sancire la sua definitiva condanna. Per contro, la protagonista de L'innocenza risorta cioè $S$. Idda, tragicommedia rappresentata al

(24) «Une solution baroque» la definisce D. DALLA VALLE in Autour de "Sigismond", cit., p. 191.

(25) Vende ou le triomfe et le sacrifice de la chasteté, cit. argument du cinquiesme acte.

(26) «Alors, levant les mains et les yeux en haut d'une manière supliante, et regardant ensuite vers les ondes, Dieu du Vistulle (s'écria-t-elle) reçois un corps pur et innocent, pour la reparation des foiblesses de mon ame, et partage avec les autres
Dieux le sacrifice de ma vie». Venda Reine de Pologne ou l'bistoire galante et curieuse de ce qui s'est passé de plus memorable en ce tems-là, La Haye, Abraham Troyel, 1705, p. 2.

(27) Cfr. P.-A. SigAL, L'bomme et le miracle dans la France médiévale (XI'-XII ${ }^{e}$ siècle), Paris, Les Éditions du Cerf, 1985, p. 268. 84. 
seminario romano nel 1662 e nel 1666, sopravvive al salto «per aiuto del Cielo» e, pur riconosciuta innocente, si ritira nella selva per dedicarsi alla preghiera ${ }^{29}$.

La morte di Vanda nel teatro di collegio richiama alla mente il tragico finale del Torrismondo tassiano, ma la follia o il suicidio per disperazione amorosa erano ricorrenti nel teatro francese $e^{30}$ : il suicidio tragicomico avviene assumendo veleno, col pugnale o per annegamento, e la prossimità della morte costituisce uno dei dati essenziali per la definizione del tragico tragicomico. Che la morte prenda la forma di guerra, duello o supplizio, che resti solo una minaccia per l'eroe e si attualizzi invece nella figura del nemico, essa caratterizza comunque la tragicommedia e la tragedia irregolare, e questa equivalenza rimanda la distinzione di genere allo scioglimento ${ }^{31}$. Nel Sigismond di Gillet la regina non si uccide; propone invece il trono polacco al suo salvatore: l'ipotesi del matrimonio è solo sfiorata, dal momento che il voto di castità lo renderebbe impossibile, ma il rifiuto non sembra definitivo e l'ipotesi resta aperta. Il lieto fine, a costo di modificare l'impianto originario della fonte, diviene elemento costitutivo della tragicommedia per distinguerla dalla tragedia, in cui - nella lettura di M. Lombardi - il fallimento di Eros segnava una disarmonia nell'universo e una fonte di immoralità ${ }^{32}$.

Il corredo mitico del personaggio Vanda non è certo secondario al suo successo: meglio di qualunque altra forma letteraria, il teatro rivela le modalità di appropriazione di una mitologia pagana rivisitata, che dopo il ballet de cour darà origine durante il ministero di Mazarino alle prime opere mitologiche vere e proprie ${ }^{33}$. Senza mai diventare una pièce à machines dove la mitologia assume un ruolo decorativo confondendo magico e meraviglioso, Vanda beneficia di un processo di codificazione letteraria che passa anche per il suo essere protagonista di una storia antica, in certo senso esemplare, che assurge a modello e simbolo ${ }^{34}$. Al di là del successo legato alla "polonofilia" francese secentesca, nelle sue varie declinazioni la regina che guida un esercito e poi si uccide per salvare il suo paese esercita una fascinazione legata alle diverse sfumature in cui si articola il tema della femminilità, tradizionalmente vincolato - almeno in letteratura - a codificazioni stereotipate. Ad esse Vanda viene ricondotta lungo le successive trasposizioni, in cui la dimensione amorosa prende il sopravvento trasformando l'eroina casta in donna innamorata, talvolta vittima del suo amore. Nel corso della metamorfosi, tuttavia, si possono rintracciare le coordinate di una «mythique de la femme» che secondo Paul Fauchery sempre anima le figure femmini-

(29) Lo scenario è analizzato da B. FILIPPI, op. cit., pp. 278 e 308: S. Idda, sposa di un principe svedese, espone al sole i suoi gioielli, ma un corvo le ruba la fede nuziale. Il primo ministro Ildoro lo trova durante una battuta di caccia e lo mette al dito. Ergasto lo riconosce e convince il principe che è un dono d'amore di sua moglie a Ildoro. Il principe li condanna a morte entrambi: il ministro sarà trascinato da un cavallo, Idda sarà gettata da una torre. Ma lei resta incolume, e la sua innocenza è riconosciuta. Tuttavia sceglie di non tornare a corte, per vivere nella selva in preghiera.

(30) Nell'Aminta del Tasso l'eroe disperato per la freddezza di Silvia si lancia da un precipizio, e i cespugli attutiscono la sua caduta. I suoi emuli tragicomici spesso sfuggono provvidenzialmente alla morte, come il mancato suicidio per annegamento di Caliris (L'Inconstance punie di La Croix) e quello di Damon (in Madont) salvato dai pescatori. Altri suicidi, invece, riescono: in Tyr et Sydon la nutrice racconta come Cassandre, vedendosi rifiutata da Belcar, si pugnali prima di gettarsi in mare dalla nave su cui era fuggita. Cfr. R. GuichemerRe, $\mathrm{La}$ tragi-comédie, Paris, PUF, 1981, p. 131.

(31) H. BABy, La tragi-comédie de Corneille à Quinault, Paris, Klincksiek, 2001, p. 78.

(32) M. Lombardi, Processo al teatro. La tragicommedia barocca e i suoi mostri, Firenze, Pacini, 1995: «L'ostracismo del dio amore dal consesso civile si accompagna alla volontà di cacciare dalle città e dalle corti gli attori che di Cupido sembrano essere i sacerdoti per il fascino che esercitano sul pubblico. Difendere Eros significa dunque salvare il teatro e la sua funzione morale e sociale. Mettere in scena un universo tragicomico e pastorale retto da Eros significa dimostrare con prove oculari (il teatro riceve l'eredità della retorica giudiziaria) la felicità, l'armonia, il trionfo di un regno retto dal Sovrano d'Amore» (p. 10).

(33) Cfr. C. Delmas, Mythologie et mythe dans le theatre français (1650-1676), Genève, Droz, 1985.

(34) M. Eliade, Mythes, rêves et mystères, Paris, Gallimard, 1957, p. 22. 
li: stadio ultimo dell'epos, la forma narrativa non rompe con i miti primordiali, anche se la mitologia soggiacente in cui si riconoscono le divinità simboliche del desiderio, della fecondità, della seduzione, della saggezza o della verginità senza macchia rislta marginale rispetto alla rielaborazione scritta ${ }^{35}$. Nel rapporto tra scrittura e mito, che apre orizzonti imprevedibili per l'intrinseca necessità di chiudere in un cronotopo qualcosa che era sospeso nello Spazio e nel tempo ${ }^{36}$, Vanda è tanti personaggi diversi, ricreati dalle opere che le hanno dato forma e riconducibili ad almeno tre grandi archetipi: la vestale depositaria del rapporto col divino, la guerriera che combatte per il suo regno, la fata delle acque che torna al suo elemento naturale.

Nella tragedia di collegio del 1644 (Vende ou le triomfe et le sacrifice de la chasteté) la dimensione eroica non si manifesta soltanto come passiva rinuncia alla vita. Vende e la «Princesse sa favorite» - recita l'argument del quinto atto - scendono in campo a combattere travestite da uomini: «enfin ces Amazones paroissent armées pour le Combat, ayant desguisé leur sexe», e alla morte della regina «la Vistule d'horreur retourne vers sa source». Alla figura di vittima sacrificale si sovrappone la donna guerriera, che infine torna all'acqua, fonte di purezza originaria. Anche senza avere a disposizione l'intero testo, è possibile individuare subito i tratti caratteristici che accompagnano il personaggio. La vicenda, pur costruita intorno al dramma dinastico legato al tema dei fratelli nemici proprio della tragedia classica (Sofocle), ripropone e rivisita l'archetipo collettivo della donna selvaggia, che gioca un ruolo importante nella mitologia slava ${ }^{37}$. Ma, incrociando la mitologia russa ed est-europea con quella indiana e orientale, è stato ipotizzato un legame tra la principessa annegata nella Vistola e il mito di Melusina. Lo spirito protettore di Vanda, dicono le leggende polacche, compare nelle vesti di una dama bianca sulle torri della capitale in caso di minaccia al paese, lanciando alte 《grida di Melusina» ${ }^{38}$. La regina scompare tra $\mathrm{i}$ flutti per disperazione, e il motivo dell'«effusion en eau» (trasformazione in acqua) appartiene al linguaggio mitico insieme a quello della formazione di fiumi e laghi per accumulo delle lacrime di un essere infelice che deve ritornare all'elemento primigenio, temporaneamente abbandonato per assumere forma umana. Lo stemma della capitale polacca presenta ancora una figura di sirena, e una ninfa della Vistola viveva fino a metà Ottocento nella tradizione orale del paese, ricca di favole sulle donne marine. La derivazione della vita dall'acqua, d'altronde, è forse la più antica delle spiegazioni scientifiche, e uno dei più remoti miti dell'umanità, nutrito di avvistamenti di creature marine che a lungo alimentarono il dibattito sulle origini ${ }^{39}$. Appare fugacemente la leggenda della donna pesce che rinuncia alla doppia natura per amore di un essere umano: Melusina, la fata-serpente antenata di Ondina, perde la voce, anima del suo incanto e della sua vitalit $a^{40}$; Vanda perde sempre la vita, finché nella nouvelle galante del 1705 non viene ripescata e nascosta dal prete del tempio affinché possa coronare il suo sogno d'amore. Nel frattempo, infatti, la vestale guerriera è diventata una donna innamorata, che con Melusina - evocata e subito rimossa - ha in comune solo l'appartenenza a un altro mondo e il congiungimento con un mortale. Le differenti caratterizzazioni francesi della regina sottolineano il suo essere diversa,

(35) FAUCHERY si riferisce nella sua analisi al romanzo del Settecento, ma ci sembra che il discorso generale sulla «mythique de la femme» possa essere applicato alla specificità del nostro personaggio. P. FAUCHERY, La destinée féminine dans le roman européen du XVIII siècle, Paris, Colin, 1972, pp. 53-55.

(36) Cfr. M. Detienne (éd.), Transcrire les mythologies, Paris, Albin Michel, 1994.

(37) Cfr. P. SAmuel, Amazones, guerrières et gail- lardes, Bruxelles, Complexe-PUG, 1975.

(38) In J. Karlowicz, La belle Mélusine et la reine, Vanda, «Archiv für Slavische Philologie», II, Berlin, 1877, pp. 594-609.

(39) A. GERBI, La disputa del Nuovo Mondo. Storia di una polemica (1750-1900), Milano-Napoli, Ricciardi, 1955.

(40) C. Jamain, La voix de Mélusine, in Mélusine moderne et contemporaine, c.d. A. Bouloumié, Angers, L'Àge d'homme, 2001, pp. 317-325. 
migliore e protettrice: è depositaria di virtù, affronta con determinazione il negativo (guerra, vendetta, morte), possiede una fragilità che la rende capace di sofferenza e mediazione. In tal senso, il matrimonio conclusivo, più che un sacrificio simbolico - come ipotizzato da F. Rosset - pare configurarsi come un recupero della dimensione umana, conseguenza dell'abbandono di quella soprannaturale. D'altronde le fate hanno, nel mito, una natura ambigua, sono dame cortesi che appartengono a un altro mondo, ma dominano il tempo e presentano tratti ricorrenti di eccezionale bellezza, ricchezza e saggezza. Melusina muta in serpente perché figlia di un essere soprannaturale e di un umano: può condurre una vita normale ma paga uno scotto al soprannaturale con la metamorfosi. Vanda, a suo modo, paga un tributo al mito che l'ha resa famosa trasformandosi in personaggio assoggettato alle regole letterarie, diventa una chiave di dibattito su questioni politiche, filosofiche ed estetiche e anche - molto più prosaicamente - un modello di riferimento per la reggenza di Anna d'Austria, come lascia intendere un passo dell'Epître nella tragicommedia di Gillet ${ }^{41}$. È quindi un personaggio mitico per gli elementi che ne compongono la figura in una situazione limite articolata intorno ad un conflitto, che si allarga dall'individuo nella sua specificità a un intero gruppo, di cui esprime quella che P. Brunel definisce la «constellation mentale» ${ }^{42}$.

SIMONA MUNARI

(41) «Aussi ne gouverna-t'elle pas long-temps, sans faire advoüer que si son sexe l'empéschoit d'estre au nombre des heros, sa vertu la mettoit au rang des plus grandes ames, et qu'enfin en un corps de femme l'on pouvoit porter un cœur veritablement masle et genereux». Gillet de la Tessonerie, Sigismond duc de Varsau, Paris, Quinet, 1646, Epître.

(42) P. Brunel, Dictionnaire des mythes littéraires, Monaco, éd. du Rocher, 1988, p. 1131. 